\title{
Effect of Metal-Assisted Catalytic Etching (MACE) on Single-Crystal Si Wafers With Faceted Macropores
}

\author{
Alexis T. Ernst ${ }^{1}$, Kurt W. Kolasinski ${ }^{2}$, Bret A. Unger ${ }^{3}$, Mark Aindow ${ }^{1}$ \\ ${ }^{1}$ Institute of Materials Science, University of Connecticut, Storrs, CT, 06269, USA \\ ${ }^{2}$ Department of Chemistry, West Chester University, West Chester, PA, 19383, USA \\ ${ }^{3}$ Department of Chemistry, University of California, Berkeley, CA, 94720, USA
}

The applications for silicon span a wide variety of fields including electronics, drug delivery, and energy storage. In particular, porous silicon (por-Si) is of great interest in optoelectronic and energy-harvesting applications due to the ability to absorb and scatter light. Additionally, Si possesses the greatest specific capacity to alloy with lithium making this element favorable for battery applications [1]. A common method used to create por-Si is metal-assisted catalytic etching (MACE), which exploits the enhanced kinetics of electron transfer at electrolyte/metal interfaces [2]. This method promotes both local and remote etching that are dependent on reaction conditions such as the $\mathrm{HF}: \mathrm{H}_{2} \mathrm{O}_{2}$ ratio, the catalyst, and the geometry $[3,4]$.

Here we consider Si wafer substrates in three crystallographic orientations: (100), (110), and (111). Prime grade silicon wafers of $500 \mu \mathrm{m}$ thickness were ablated using a Spectra-Physics Quanta Ray INDIHG- $205 \mathrm{Nd}$ :YAG laser. Following ablation, wafers were chemically etched using $\mathrm{KOH}(\mathrm{aq})$ solution to create crystallographically-defined macropores. MACE was achieved using Ag nanoparticles as the catalyst with a $\mathrm{HF}+\mathrm{H}_{2} \mathrm{O}_{2}$ solution as the etchant. The wafers were examined in two conditions: after chemical etching and after subsequent MACE etching. These studies were used to reveal the size distribution, morphology and crystallographic orientations of the etch track pores.

Figures 1A-1F are secondary electron SEM images that show the overall morphology of the chemicallyetched macropores, and the fibrous topography of the MACE-etched surfaces. These latter surfaces are consistent with the interconnected Si nanowire morphologies reported elsewhere [5]. The details of these structures were investigated using FIB-cut cross-sectional TEM specimens prepared in a dual-beam FEI Helios NanoLab 460F1 Ga ${ }^{+}$FIB-SEM. Sequential e-beam and ion-beam Pt deposition was used to protect the delicate porous surface features. Examples of BF and HAADF STEM data obtained for one such specimen from a MACE-etched (110) Si-wafer are shown in Figures 2. These data were obtained using a Talos F200X operating at an accelerating voltage of $200 \mathrm{kV}$. Such images reveal the orientations of the etch-track pores and the presence of $\mathrm{Ag}$ nanoparticles at the tips of the etch tracks.

Reference:

[1] L Mai, X Tian, X Xu, L Chang, and L. Xu, Chem. Rev. 114 (2014), pp. 11828-11862

[2] X Li, Curr. Opin. Solid State Mater. Sci. 16:2 (2012) pp. 71-81

[3] C Chartier, S Bastide, and C Lévy-Clément. Electrochimica Acta. 53:17 (2008), pp. 5509-5516

[4] C Chiappini, X Liu, JR Fakhoury, and M Ferrari. Adv. Func. Mater. 20 (2010), pp. 2231-2239

[5] KW Kolasinski, BA Unger, A Ernst, M Aindow, Front Chem. 6 (2019) art. \#651

[6] Funding was provided by NSF Grant \#1825331, West Chester University, and the resources of the Center for Microanalysis and Imaging Research and Training (CMIRT)

[7] The microscopy studies were performed in the UConn / Thermo Fisher Scientific Center for Advanced Microscopy and Materials Analysis (CAMMA). 

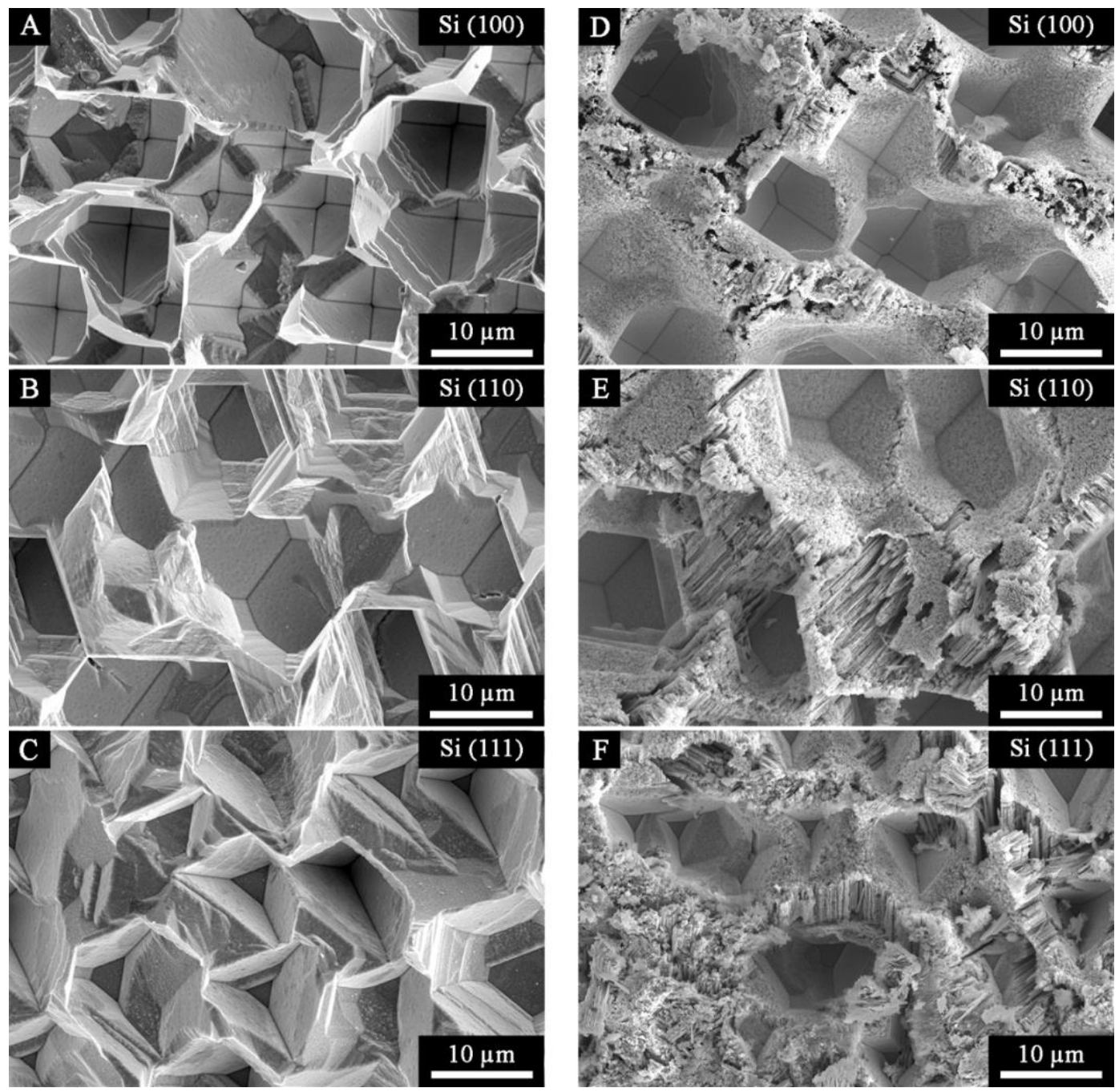

Figure 1. Comparison of surface topography before and after MACE on Si wafers (100), (110) and (111). A)-C) are chemically etched macropores. D)-F) are MACE etched macropores.
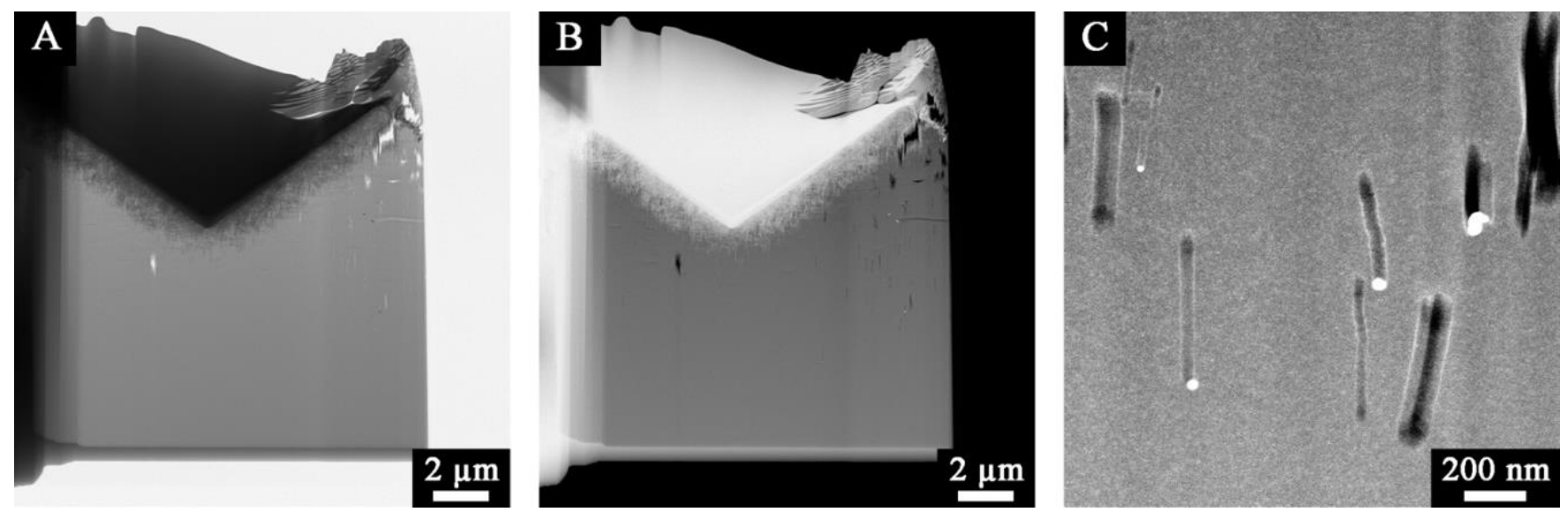

Figure 2. Cross-sectional STEM data from a MACE-etched (110) Si wafer with macropores. A) BF image. B,C) HAADF STEM images. The image in (C) reveals Ag nanoparticles at the tips of etch track pores. 